\title{
Numerical Simulation of Anisotropic Mean Curvature of Graphs in Relative Geometry
}

\section{Dieu Hung Hoang, Michal Beneš, Tomáš Oberhuber}

Department of Mathematics

Faculty of Nuclear Sciences and Physical Engineering

Czech Technical University in Prague

Trojanova 13, 12000 Praha, Czech Republic

hoangdieu@fjfi.cvut.cz, michal.benes@fjfi.cvut.cz, tomas.oberhuber@fjfi.cvut.cz

\begin{abstract}
The aim of this paper is the numerical simulation of anisotropic mean curvature of graphs in the context of relative geometry, developed in [1]. We extend results in [4] to our problem; we prove an existence theorem and energy equality. The numerical scheme is based on the method of lines where the spatial derivatives are approximated by finite differences [2]. We then solve the resulting ODE system by means of the adaptive RungeKutta-Merson method. To show the stability of the scheme we prove the discrete version of the energy equality. Finally, we show experimental order of convergence and results of numerical experiments with various anisotropy settings.
\end{abstract}

Keywords: anisotropy; mean curvature; Finsler geometry; method of lines; FDM

\section{Introduction}

The paper studies the following motion law for surfaces in $\mathbb{R}^{3}$ denoted by $\Gamma$ :

velocity $=$ curvature + forcing

in a certain sense which is specified below. Both the velocity and the curvature are evaluated with respect to the direction given by a vector locally influenced by the orientation of the Euclidean normal vector to $\Gamma$.

One example of the law (1) is represented by the isotropic mean-curvature flow given by the equation

$v_{\Gamma}=-\kappa_{\Gamma}+f$ on $\Gamma(t)$

in the direction of $n_{\Gamma}$ which is the Euclidean normal vector to $\Gamma$, while $v_{\Gamma}$ the normal velocity, $\kappa_{\Gamma}$ the mean curvature, and $f$ the forcing term. The equation (2) in the form of the Gibbs-Thompson law is contained in the modified Stefan problem. For details, we refer the reader to $[9,16]$. 
One of few anisotropic examples where the analytical solution is known considers a ball under the relative geometry which shrinks according to (1) with $f=0$. In this case we have the initial ball with radius $r_{0}$, normal velocity $\dot{r}$, actual curvature along the ball of radius $r$ being $\frac{1}{r}$. The equation (1) reads

$\dot{r}=-\frac{1}{r}$

and has the solution

$r(t)=\sqrt{r_{0}^{2}-2 t}$

This law has been intensively studied, see e.g. [4, 5, 13].

This paper deals with the motion by anisotropic mean curvature in relative geometry associated with the Finsler metric, developed in [1], which reads

$v_{\Gamma, \phi}=-\kappa_{\Gamma, \phi}+f$ on $\Gamma(t)$

Here, $v_{\Gamma, \phi}$ denotes the normal velocity, $\kappa_{\Gamma, \phi}$ is the anisotropic mean curvature of $\Gamma(t)$ with respect to the Finsler metric $\phi$, and $f$ is the forcing term.

Deckelnick and Dziuk proved the convergence and gave the optimal error estimates using finite element method for graph [4, 7] and parametric case [8]. Haußer and Voigt [11] presented a parametric finite element approximation for a regularized version. Pozzi studied the anisotropic mean curvature flow in higher codimension in [15].

\section{Anisotropy in Relative Geometry}

In what follows we shall first define anisotropy by means of the Finsler geometry; then, we shall transform the motion law (3) into graph formulation. For this purpose, we assume that there is a smooth function with non-vanishing gradient $p: \mathbb{R}^{2+1} \rightarrow \mathbb{R}$ such that

$$
\Gamma(t)=\left\{[x, y] \in \mathbb{R}^{3} \mid y=p(t, x), x \in \Omega \subset \mathbb{R}^{2}\right\} .
$$

We say that a continuous function $\phi: \mathbb{R}^{3} \rightarrow \mathbb{R}_{0}^{+}$is a Finsler metric if it satisfies the properties

1. $\phi \in C^{3+\alpha}(\mathbb{R}-\{0\})$,

2. $\phi^{2}$ is strictly convex,

3. $\phi(t \eta)=|t| \phi(\eta), t \in \mathbb{R}, \eta \in \mathbb{R}^{3}$,

4. $\lambda|\eta| \leq \phi(\eta) \leq \Lambda|\eta|, \eta \in \mathbb{R}^{3}$,

for two suitable constants $0<\lambda \leq \Lambda<\infty$.

Associated to $\phi$ we define the unit ball (also so-called Wulff shape) 
$B_{\phi}=\left\{\eta \in \mathbb{R}^{3} \mid \phi(\eta) \leq 1\right\}$.

One can prove that a dual function $\phi^{0}: \mathbb{R}^{3} \rightarrow \mathbb{R}_{0}^{+}$given by

$\phi^{0}\left(\eta^{*}\right)=\sup \left\{\eta^{*} \cdot \eta \mid \eta \in B_{\phi}\right\}$

is also a Finsler metric.

For simplicity we use $\eta$ instead of $\eta^{*}$. Then the following relations hold [3]

$\phi_{\eta}^{0}(t \eta)=\frac{t}{|t|} \phi_{\eta}^{0}(\eta), \quad \phi_{\eta \eta}^{0}(t \eta)=\frac{1}{|t|} \phi_{\eta \eta}^{0}(\eta), \quad t \in \mathbb{R}-\{0\}$,

$\zeta \cdot \phi^{0}(\eta) \phi_{\eta \eta}^{0}(\eta) \cdot \zeta \geq \gamma_{0}\left|\zeta-\frac{\zeta \cdot \eta}{|\eta|^{2}} \eta\right|^{2}, \quad \eta \neq 0, \quad \zeta \in \mathbb{R}^{3}, \quad \gamma_{0}>0$,

where the index $\eta$ means the derivative with respect to $\eta$.

We define the map $T^{0}: \mathbb{R}^{3} \rightarrow \mathbb{R}^{3}$ as

$T^{0}(\eta)=\left(\tilde{T}^{0}(\eta), T_{3}^{0}(\eta)\right)=\phi^{0}(\eta) \phi_{\eta}^{0}(\eta), \quad \eta \neq 0$,

$T^{0}(0)=0$.

Then, the $\phi$-normal vector, $\phi$-mean curvature, and $\phi$-normal velocity of $\Gamma$ are defined as

$n_{\Gamma, \phi}=\frac{T^{0}(\nabla p,-1)}{\phi^{0}(\nabla p,-1)}=\phi_{\eta}^{0}(\nabla p,-1)$,

$\kappa_{\Gamma, \phi}=\operatorname{div} n_{\Gamma, \phi}=\nabla \cdot \frac{\widetilde{T}^{0}(\nabla p,-1)}{\phi^{0}(\nabla p,-1)^{\prime}}$

$v_{\Gamma, \phi}=-\frac{\partial_{t} p}{\phi^{0}(\nabla p,-1)}$.

By substituting the quantities (4)-(6) into the Eq. (3), we obtain the non-linear parabolic partial differential equation

$\partial_{t} p=\phi^{0}(\nabla p,-1)\left(\nabla \cdot\left(\frac{\tilde{T}^{0}(\nabla p,-1)}{\phi^{0}(\nabla p,-1)}\right)+f\right)$ on $\Omega \times(0, T)$.

The initial and boundary conditions are given by

$\left.p\right|_{t=0}=p_{0}$ on $\bar{\Omega}$,

$p=p_{0}$ on $\partial \Omega \times(0, T)$.

In our numerical experiments we use the Finsler metrics listed below. We denote $n=\frac{\eta}{|\eta|}$. The corresponding Wulff shapes are illustrated in Fig. 1. 
The 4-fold anisotropy reads as

$\phi(\eta)=|\eta|\left(1-A_{1}\left(1-\left(n_{1}^{4}+n_{2}^{4}+n_{3}^{4}\right)\right)\right)$.

The 6-fold anisotropy reads as

$$
\begin{aligned}
\phi_{\eta}^{0}(\eta) & =|\eta|\left(1-A_{1}\left(n_{1}^{4}+n_{2}^{4}+n_{3}^{4}-\frac{3}{5}\right)\right. \\
& \left.+A_{2}\left(3\left(n_{1}^{4}+n_{2}^{4}+n_{3}^{4}\right)+66 n_{1}^{2} n_{2}^{2} n_{3}^{2}-\frac{17}{7}\right)\right)
\end{aligned}
$$

The 8-fold anisotropy reads as

$$
\begin{aligned}
\phi_{\eta}^{0}(\eta) & =|\eta|\left(1-A_{1}\left(n_{1}^{8}+n_{2}^{8}+n_{3}^{8}\right.\right. \\
& -28\left(n_{1}^{6} n_{2}^{2}+n_{1}^{2} n_{2}^{6}+n_{2}^{6} n_{3}^{2}+n_{2}^{2} n_{3}^{6}+n_{3}^{6} n_{1}^{2}+n_{3}^{2} n_{1}^{6}\right) \\
& \left.\left.+70\left(n_{1}^{4} n_{2}^{4}+n_{2}^{4} n_{3}^{4}+n_{3}^{4} n_{1}^{4}\right)\right)\right) .
\end{aligned}
$$

The regularized $l_{1}$-anisotropy reads as

$$
\phi_{\eta}^{0}(\eta)=\sum_{i=1}^{3}\left(\eta_{i}^{2}+A_{1} \sum_{j=1}^{3} \eta_{j}^{2}\right)^{\frac{1}{2}} \text {. }
$$

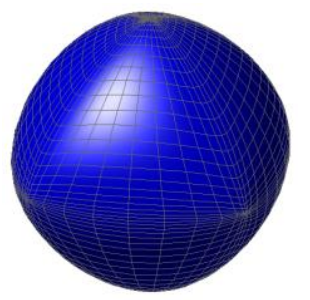

4-fold anisotropy, $A_{1}=0.24$

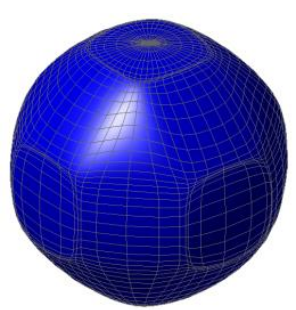

8-fold anisotropy, $A_{1}=-0.015$

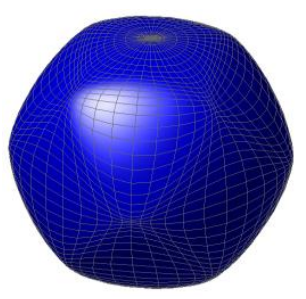

6-fold anisotropy, $A_{1}=-0.035, A_{2}=-0.035$

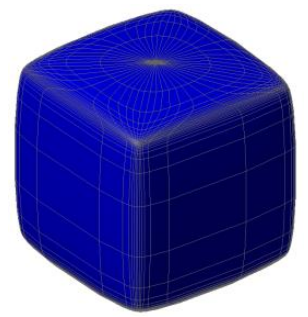

regularized $l_{1}$-norm, $A_{1}=0.01$

Figure 1

Wulff shapes for various anisotropies 


\section{Analytical Properties}

In the following section we shall introduce some analytical results for law (7) in the context of relative geometry, which are due to $[4,11,12]$. We shall prove the energy equality and give the existence result for our problem.

Theorem 1. For the solution of problem (7)-(9), one has the energy equality

$\int_{\Omega} p_{t}\left(\frac{p_{t}}{\phi^{0}(\nabla p,-1)}-f\right)+\frac{d}{d t} \int_{\Omega} \phi^{0}(\nabla p,-1)=0$.

If $f=0$, then

$\int_{\Omega} \frac{p_{t}^{2}}{\phi^{0}(\nabla p,-1)}+\frac{d}{d t} \int_{\Omega} \phi^{0}(\nabla p,-1)=0$.

Proof. Since $p_{t}=0$ on $\partial \Omega$, the proof is straightforward

$$
\begin{aligned}
\frac{d}{d t} \int_{\Omega} \phi^{0}(\nabla p,-1) & =\int_{\Omega} \phi_{\eta}^{0}(\nabla p,-1) \cdot[\nabla p,-1]_{t}=\int_{\Omega}\left[\nabla p_{t}, 0\right] \cdot \frac{T^{0}(\nabla p,-1)}{\phi^{0}(\nabla p,-1)} \\
& =\int_{\Omega} \nabla p_{t} \cdot \frac{\tilde{T}^{0}(\nabla p,-1)}{\phi^{0}(\nabla p,-1)}=\int_{\Omega} p_{t} \nabla \cdot \frac{\widetilde{T}^{0}(\nabla p,-1)}{\phi^{0}(\nabla p,-1)} \\
& =-\int_{\Omega} p_{t}\left(\frac{p_{t}}{\phi^{0}(\nabla p,-1)}-f\right) .
\end{aligned}
$$

If $f=0$, we obtain the equality (14).

Lemma 1. Let $v(\tilde{\eta})=\phi^{0}(\tilde{\eta},-1), a_{i}=\frac{T_{i}^{0}(\nabla p,-1)}{\phi^{0}(\nabla p,-1)}$, and $a_{i j}=\frac{\partial a_{i}}{\partial \eta_{j}}$. Then for the solution of the problem (7)-(9) with $f=0$ one has the identity

$$
\begin{aligned}
v_{t} & =\sum_{i, j=1}^{2} \frac{\partial}{\partial x_{i}}\left(a_{i j} \frac{\partial v}{\partial x_{j}}\right) v+\sum_{l=1}^{2} \kappa_{\phi} a_{l} \frac{\partial v}{\partial x_{l}} \\
& +\sum_{i, j, k=1}^{2} a_{i j} a_{k l} \frac{\partial^{2} p}{\partial x_{i} \partial x_{k}} \frac{\partial^{2} p}{\partial x_{j} \partial x_{l}} v .
\end{aligned}
$$

Proof. We have

$$
v_{t}=\phi_{\widetilde{\eta}}^{0}(\nabla p,-1) \nabla p_{t}=\sum_{l=1}^{2} a_{l} \frac{\partial\left(v \kappa_{\phi}\right)}{\partial x_{l}}=v \sum_{l=1}^{2} a_{l} \frac{\partial \kappa_{\phi}}{\partial x_{l}}+\kappa_{\phi} \sum_{l=1}^{2} a_{l} \frac{\partial v}{\partial x_{l}}
$$


Let now compute

$$
\begin{aligned}
\sum_{l=1}^{2} a_{l} \frac{\partial \kappa_{\phi}}{\partial x_{l}} & =\sum_{l=1}^{2} a_{l} \frac{\partial}{\partial x_{l}} \sum_{i=1}^{2} \frac{\partial}{\partial x_{i}} a_{i}=\sum_{i, l=1}^{2} a_{l} \frac{\partial}{\partial x_{i}} \frac{\partial}{\partial x_{l}} a_{i} \\
& =\sum_{i, l=1}^{2} \frac{\partial}{\partial x_{i}}\left(a_{l} \frac{\partial}{\partial x_{l}} a_{i}\right)-\sum_{i, l=1}^{2} \frac{\partial}{\partial x_{i}} a_{l} \frac{\partial}{\partial x_{l}} a_{i}
\end{aligned}
$$

Since

$\frac{\partial}{\partial x_{j}} v=\sum_{k=1}^{2} a_{k} \frac{\partial}{\partial x_{j}} \frac{\partial}{\partial x_{k}} p, \quad \frac{\partial}{\partial x_{l}} a_{i}=\sum_{j=1}^{2} a_{i j} \frac{\partial}{\partial x_{j}} \frac{\partial}{\partial x_{l}} p$,

we get the identity (15).

Theorem 2. Let $\partial \Omega \in C^{3+\alpha}$ and $d(x):=\operatorname{dist}(x, \partial \Omega)$. We assume that

$\sum_{i, j=1}^{2} \phi_{\eta_{i} \eta_{j}}^{0}(\nabla d(x), 0) d_{x_{i} x_{j}}(x) \leq 0, x \in \partial \Omega$.

Let $p_{0} \in C^{3+\alpha}(\bar{\Omega})$ satisfies the compatibility condition

$\sum_{i, j=1}^{2} \phi_{\eta_{i} \eta_{j}}^{0}\left(\nabla p_{0},-1\right) p_{0, x_{i} x_{j}}=0, \quad x \in \partial \Omega$.

Then (7)-(9) with $f=0$ has a solution $p \in H^{3+\alpha, \frac{3+\alpha}{2}}(\bar{\Omega} \times[0, T])$ with $p_{t} \in$ $L^{2}\left(0, T ; H^{2,2}(\Omega)\right)$ for all $T<\infty$.

Proof. Similarly as in [4] we are looking for a solution of the initial boundary value problem

$p_{t}-\sum_{i, j=1}^{2} a_{i j}(\nabla p) p_{x_{i}} p_{x_{j}}=0$

but with the difference

$a_{i j}(\tilde{\eta})=\phi^{0}(\tilde{\eta},-1) \phi_{\eta_{i} \eta_{j}}^{0}(\tilde{\eta},-1)$.

Since $\phi^{0}$ is a Finsler metric, $\phi^{0} \in C^{0,1}(\mathbb{R})$ holds. Moreover, since $\phi^{0} \in$ $C^{3+\alpha}(\mathbb{R}-\{0\})$, we have $a_{i j} \in C^{0,1}(\mathbb{R})$. 
Following standard lines of Theorem 4.1 in [4] and using the previous Lemma 1 we can show there is a constant $K$ such that for every solution $p^{\tau}$ of

$$
\begin{aligned}
p_{t}^{\tau}-\tau \sum_{i, j=1}^{2} a_{i j}\left(\nabla p^{\tau}\right) p_{x_{i} x_{j}}^{\tau}-(1-\tau) \Delta p^{\tau} & =0 & & \text { in } \Omega \times(0, T), \\
p^{\tau} & =\tau p_{0} & & \text { on } \partial \Omega \times(0, T), \\
p^{\tau}(\cdot, 0) & =\tau p_{0} & & \text { on } \Omega,
\end{aligned}
$$

the estimate

$$
\max _{\Omega \times(0, T)}\left|p^{\tau}\right|+\max _{\Omega \times(0, T)}\left|\nabla p^{\tau}\right| \leq K
$$

is valid. This means (7)-(9) with $f=0$ has a solution $p \in H^{3+\alpha, \frac{3+\alpha}{2}}(\bar{\Omega} \times[0, T])$.

\section{Numerical Scheme}

We employed the numerical scheme based on the method of lines. The spatial derivatives are discretized and the time variable is left continuous. After discretizing the problem by finite differences in space, we solve the resulting ODE system by the adaptive Runge-Kutta-Merson method. We consider the computational domain $\Omega=\left(0, L_{1}\right) \times\left(0, L_{2}\right)$ and introduce the following notation:

$$
\begin{aligned}
h_{1} & =\frac{L_{1}}{N_{1}}, h_{2}=\frac{L_{2}}{N_{2}} \\
\omega_{h} & =\left\{\left[i h_{1}, j h_{2}\right] \mid i=1, \cdots, N_{1}-1 ; j=1, \cdots, N_{2}-1\right\}, \\
\bar{\omega}_{h} & =\left\{\left[i h_{1}, j h_{2}\right] \mid i=0, \cdots, N_{1} ; j=0, \cdots, N_{2}\right\}, \\
\gamma_{h} & =\omega_{h}-\bar{\omega}_{h}, \\
u_{i j} & =u\left(i h_{1}, j h_{2}\right), \\
u_{x_{1}, i j} & =\frac{u_{i+1, j}-u_{i j}}{h_{1}}, u_{x_{2}, i j}=\frac{u_{i, j+1}-u_{i j}}{h_{2}}, \\
u_{\bar{x}_{1}, i j} & =\frac{u_{i j}-u_{i-1, j}}{h_{1}}, u_{\bar{x}_{2}, i j}=\frac{u_{i j}-u_{i, j-1}}{h_{2}}, \\
\nabla_{h} u & =\left[u_{x_{1}}, u_{x_{2}}\right], \\
\bar{\nabla}_{h} u & =\left[u_{\bar{x}_{1}}, u_{\bar{x}_{2}}\right], \\
\mathcal{P}_{h} g & =\left.g\right|_{\bar{\omega}_{h}} .
\end{aligned}
$$


We define the following expressions

$$
\begin{aligned}
(f, g)_{h} & =\sum_{i=1}^{N_{1}-1} \sum_{j=1}^{N_{2}-1} h_{1} h_{2} f_{i j} g_{i j}, \quad\|f\|_{h}^{2}=(f, f)_{h}, \\
\left(f^{1}, g^{1}\right\rfloor & =\sum_{i=1}^{N_{1}} \sum_{j=1}^{N_{2}-1} h_{1} h_{2} f_{i j}^{1} g_{i j}^{1}, \\
\left(f^{2}, g^{2}\right] & =\sum_{i=1}^{N_{1}-1} \sum_{j=1}^{N_{2}} h_{1} h_{2} f_{i j}^{2} g_{i j}^{2}, \\
(\boldsymbol{f}, \boldsymbol{g}] & =\left(f^{1}, g^{1}\right\rfloor+\left(f^{2}, g^{2} l,\right. \\
(f, g] & =\sum_{i=1}^{N_{1}} \sum_{j=1}^{N_{2}} h_{1} h_{2} f_{i j} g_{i j} .
\end{aligned}
$$

We then propose a semi-discrete scheme [2]

$$
\begin{aligned}
p_{t}^{h} & =\phi^{0}\left(\bar{\nabla}_{h} p^{h},-1\right)\left(\nabla_{h} \cdot\left(\frac{\tilde{T}^{0}\left(\bar{\nabla}_{h} p^{h},-1\right)}{\phi^{0}\left(\bar{\nabla}_{h} p^{h},-1\right)}\right)+f\right) \text { on } \omega_{h} \times(0, T), \\
\left.p^{h}\right|_{t=0} & =\mathcal{P}_{h} p_{0} \text { on } \bar{\omega}_{h} \\
p^{h} & =\mathcal{P}_{h} p_{0} \text { on } \gamma_{h} \times(0, T) .
\end{aligned}
$$

This is an ODE system and existence and uniqueness of solutions are guaranteed by the theory of ordinary differential equations (the Picard-Lindelöf theorem).

As the stability criterion we use the basic energy equality (14). For this purpose we shall now prove the discrete version of Theorem 1 .

Theorem 3. For the solution of problem (16), the following energy equality holds

$$
\left(p_{t}^{h}, \frac{p_{t}^{h}}{\phi^{0}\left(\bar{\nabla}_{h} p^{h},-1\right)}-f\right)_{h}+\frac{d}{d t}\left(\phi^{0}\left(\bar{\nabla}_{h} p^{h},-1\right), 1\right]=0 .
$$

If $f=0$, then

$$
\left(\left(p_{t}^{h}\right)^{2}, \frac{1}{\phi^{0}\left(\bar{\nabla}_{h} p^{h},-1\right)}\right)_{h}+\frac{d}{d t}\left(\phi^{0}\left(\bar{\nabla}_{h} p^{h},-1\right), 1\right]=0 .
$$

Proof. Applying the grid version of Green's formula as in [2], we obtain

$$
\begin{aligned}
& \left(p_{t}^{h}, \frac{p_{t}^{h}}{\phi^{0}\left(\bar{\nabla}_{h} p^{h},-1\right)}-f\right)_{h}=\left(p_{t}^{h}, \nabla_{h} \cdot\left(\frac{\tilde{T}^{0}\left(\bar{\nabla}_{h} p^{h},-1\right)}{\phi^{0}\left(\bar{\nabla}_{h} p^{h},-1\right)}\right)\right)_{h} \\
& =\left(\bar{\nabla}_{\mathrm{h}} p_{t}^{h}, \frac{\tilde{T}^{0}\left(\bar{\nabla}_{h} p^{h},-1\right)}{\phi^{0}\left(\bar{\nabla}_{h} p^{h},-1\right)}\right] \\
& =-\left.\left.\sum_{i=1}^{N_{1}} \sum_{j=1}^{N_{2}-1} h_{1} h_{2} p_{t}^{h}\right|_{\bar{x}_{1}, i j} \frac{T_{1}^{0}\left(\bar{\nabla}_{h} p^{h},-1\right)}{\phi^{0}\left(\bar{\nabla}_{h} p^{h},-1\right)}\right|_{i j}
\end{aligned}
$$




$$
\begin{aligned}
& -\left.\left.\sum_{i=1}^{N_{1}-1} \sum_{j=1}^{N_{2}} h_{1} h_{2} p_{t}^{h}\right|_{\bar{x}_{2}, i j} \frac{T_{2}^{0}\left(\bar{\nabla}_{h} p^{h},-1\right)}{\phi^{0}\left(\bar{\nabla}_{h} p^{h},-1\right)}\right|_{i j} \\
& =-\left.\left.\sum_{i=1}^{N_{1}} \sum_{j=1}^{N_{2}} h_{1} h_{2} p_{t}^{h}\right|_{\bar{x}_{1}, i j} \frac{T_{1}^{0}\left(\bar{\nabla}_{h} p^{h},-1\right)}{\phi^{0}\left(\bar{\nabla}_{h} p^{h},-1\right)}\right|_{i j} \\
& -\left.\left.\sum_{i=1}^{N_{1}} \sum_{j=1}^{N_{2}} h_{1} h_{2} p_{t}^{h}\right|_{\bar{x}_{2}, i j} \frac{T_{2}^{0}\left(\bar{\nabla}_{h} p^{h},-1\right)}{\phi^{0}\left(\bar{\nabla}_{h} p^{h},-1\right)}\right|_{i j}=-\frac{d}{d t}\left(\phi^{0}\left(\bar{\nabla}_{h} p^{h},-1\right), 1\right] .
\end{aligned}
$$

If $f=0$, we get the equality (17).

\section{Computational Results}

We first investigate the convergence of the numerical scheme. Then, we explore the long time behaviour of the anisotropic motion law (3).

Experimental order of convergence. The computations have been performed over a range of different grid resolutions which allows quantifying the numerical convergence by the experimental order of convergence (EOC). A numerical solution computed on the finest grid is used to substitute the analytical solution. Given errors Error ${ }_{1}$ and Error 2 for two mesh sizes $h_{1}, h_{2}$, respectively, the EOC is defined as

$\mathrm{EOC}=\frac{\log \left(\text { Error }_{1} / \text { Error }_{2}\right)}{\log \left(\mathrm{h}_{1} / \mathrm{h}_{2}\right)}$.

The result is shown in the following table.

Table 1

Experimental order of convergence of the scheme (16)

\begin{tabular}{l|lllll}
\hline$N$ & $h$ & Error $L_{\infty}$ & EOC $L_{\infty}$ & Error $L_{2}$ & EOC $L_{2}$ \\
\hline 50 & $1 / 50$ & 0.05924 & - & 0.01175 & - \\
100 & $1 / 100$ & 0.03676 & 0.68843 & 0.00731 & 1.00000 \\
150 & $1 / 150$ & 0.02689 & 0.77219 & 0.00511 & 1.00000 \\
200 & $1 / 200$ & 0.02058 & 0.92781 & 0.00357 & 1.00000 \\
\hline
\end{tabular}

Morphology evolution. We present the solutions at different times for various anisotropies. Figs. 2-6 show surface evolutions under anisotropic mean curvature flow without the forcing term $(f=0)$. Anisotropy is shown to be crucial in the formation of different surface morphologies. The surface is first determined by symmetry of anisotropy; it then evolves towards to the flat surface. Finally, the effect of the forcing term $f$ on the surface evolution is shown in Fig. 7. 

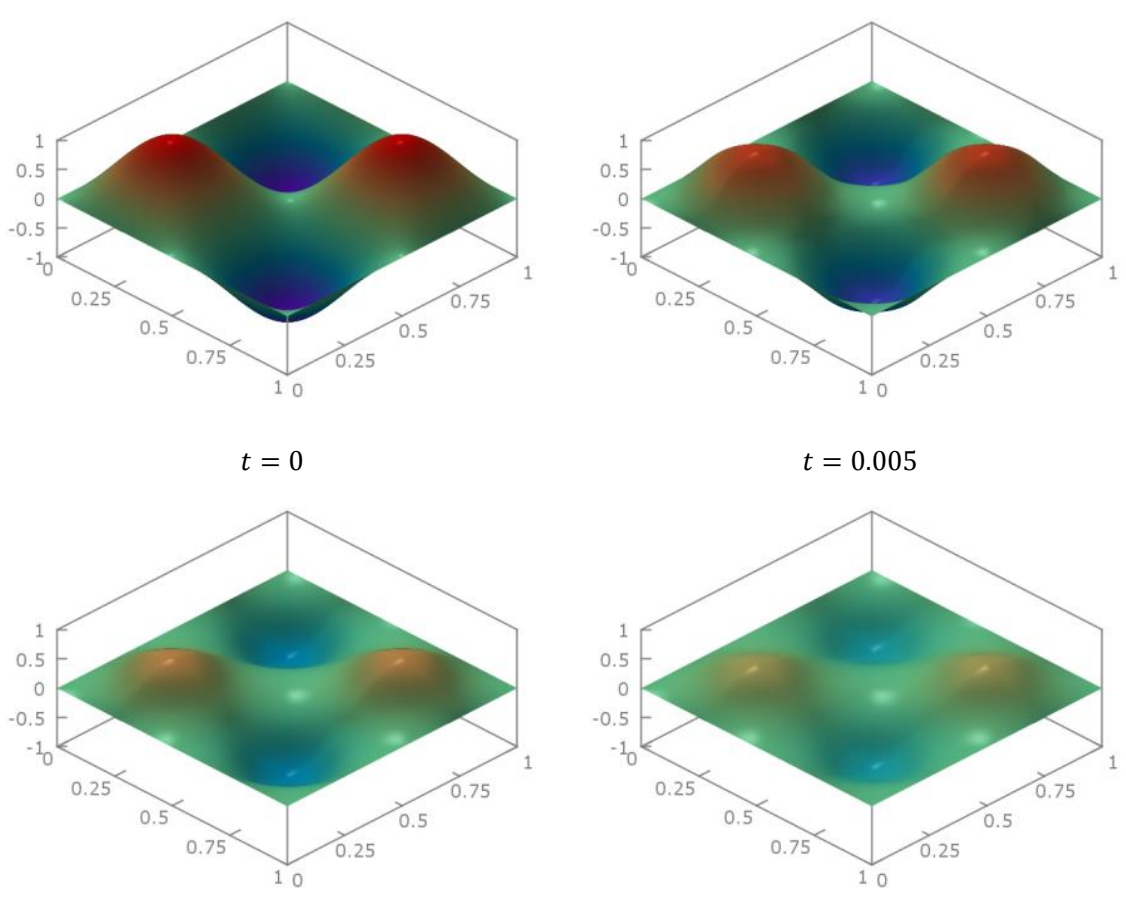

$t=0.01$

$$
t=0.015
$$
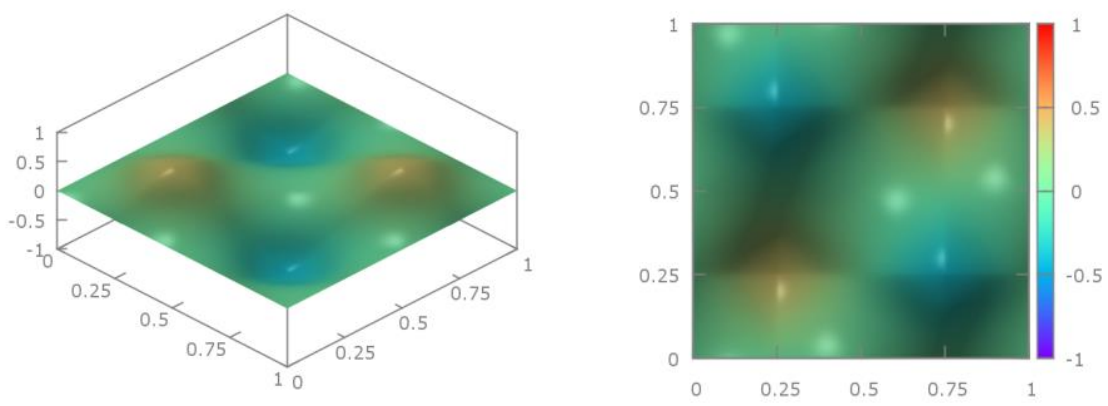

$t=0.02$

$$
t=0.02
$$

Figure 2

Morphology evolution for $f=0$, the 4-fold anisotropy (10) with $A_{1}=0.24$, $p_{0}\left(x_{1}, x_{2}\right)=\sin \left(2 x_{1}\right) \sin \left(2 x_{2}\right)$ at different times 

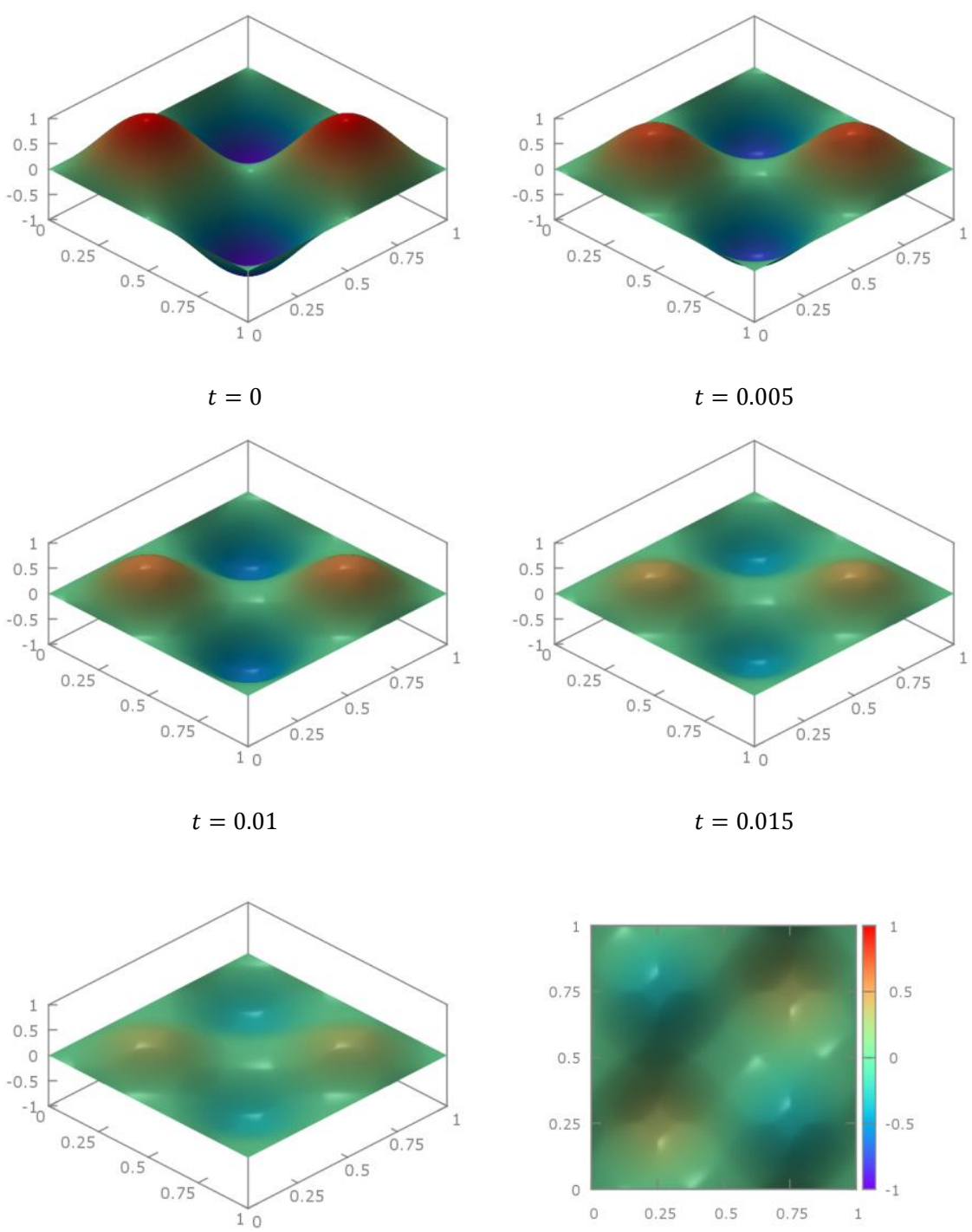

$$
t=0.02
$$

$$
t=0.02
$$

Figure 3

Morphology evolution for $f=0$, the 6-fold anisotropy (11) with $A_{1}=-0.035, A_{2}=-0.035$, $p_{0}\left(x_{1}, x_{2}\right)=\sin \left(2 x_{1}\right) \sin \left(2 x_{2}\right)$ at different times 

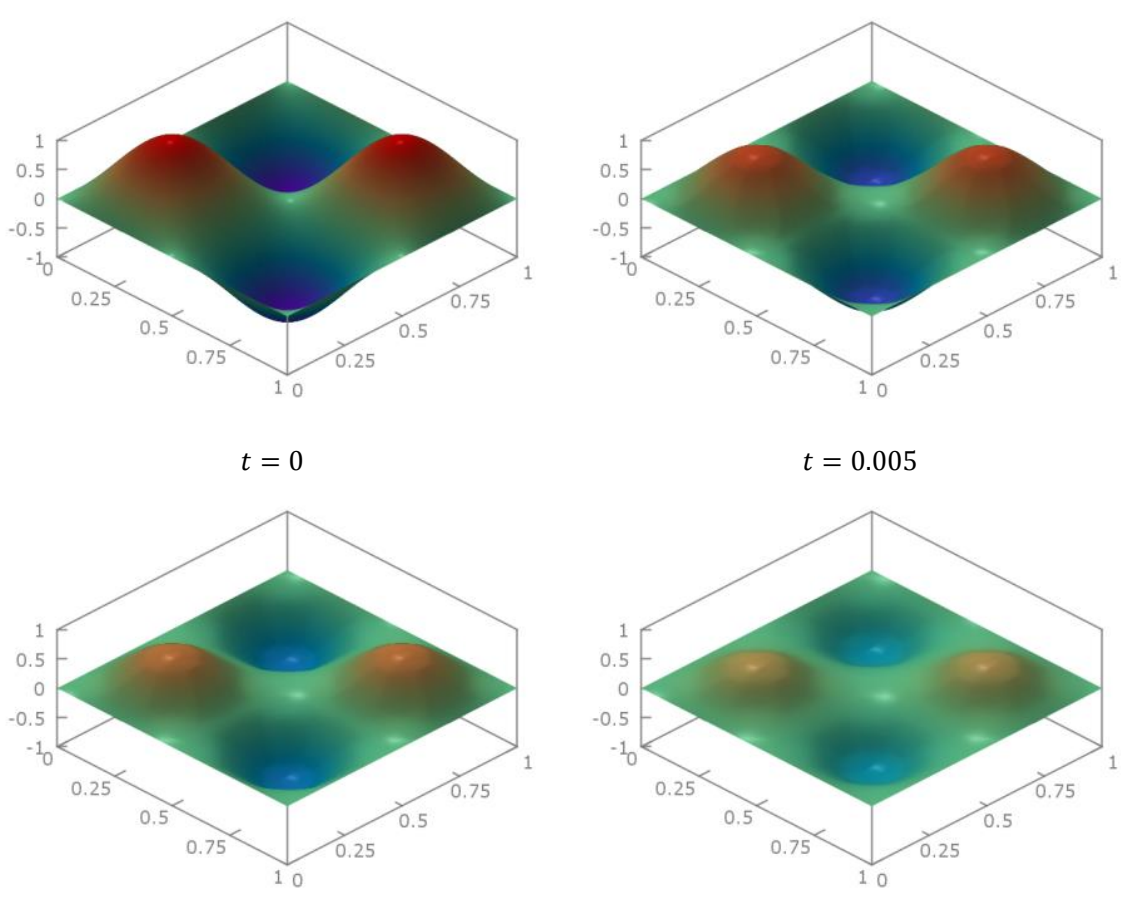

$t=0.01$

$$
t=0.015
$$
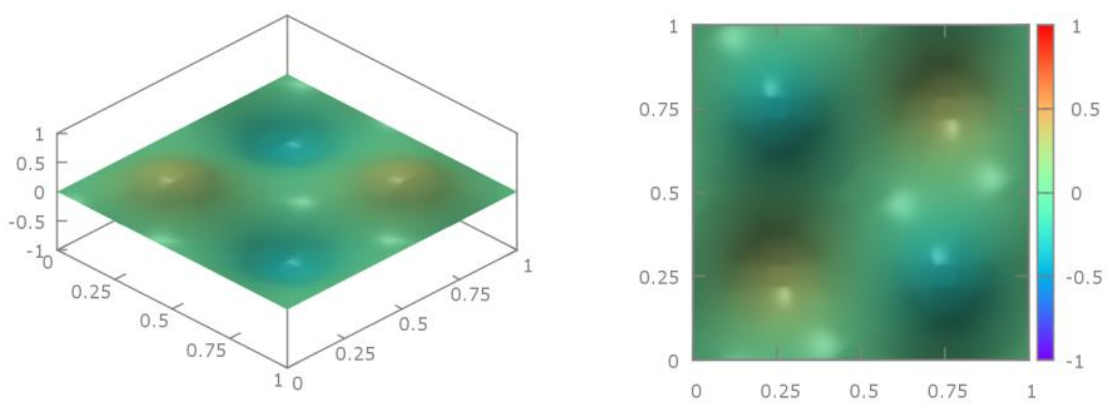

$t=0.02$

$$
t=0.02
$$

Figure 4

Morphology evolution for $f=0$, the 8 -fold anisotropy (12) with $A_{1}=-0.015$, $p_{0}\left(x_{1}, x_{2}\right)=\sin \left(2 x_{1}\right) \sin \left(2 x_{2}\right)$ at different times 

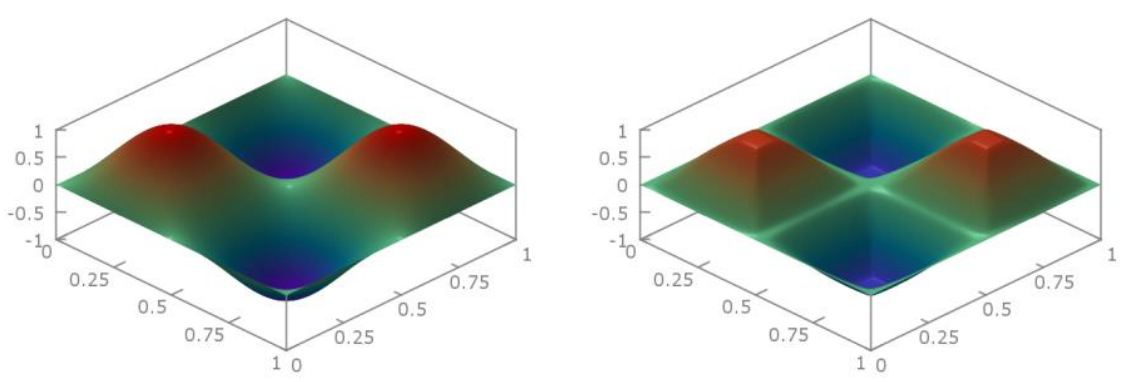

$t=0$

$t=0.005$
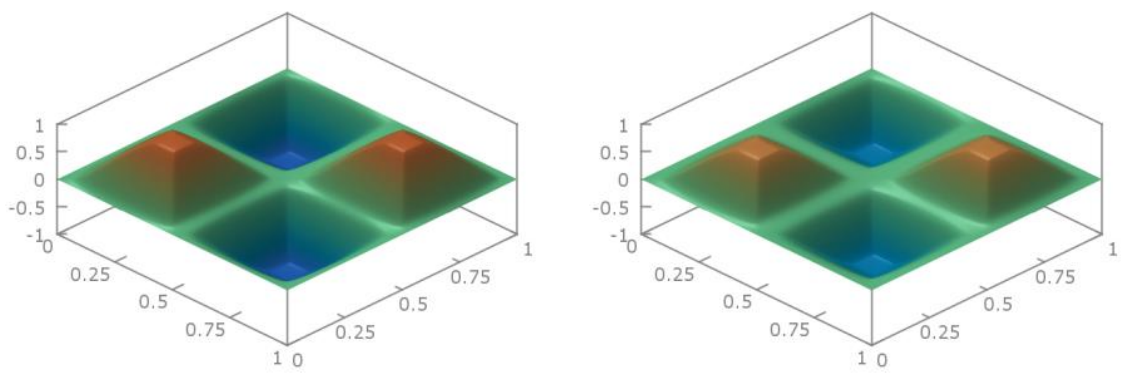

$t=0.01$

$t=0.015$
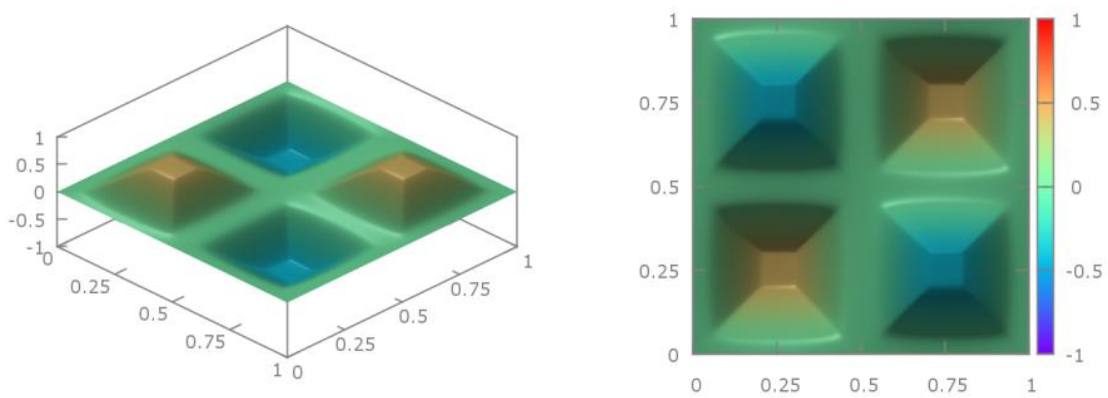

$t=0.02$

$t=0.02$

Figure 5

Morphology evolution for $f=0$, the regularized $l_{1}$ norm (13) with $A_{1}=0.01$, $p_{0}\left(x_{1}, x_{2}\right)=\sin \left(2 x_{1}\right) \sin \left(2 x_{2}\right)$ at different times 

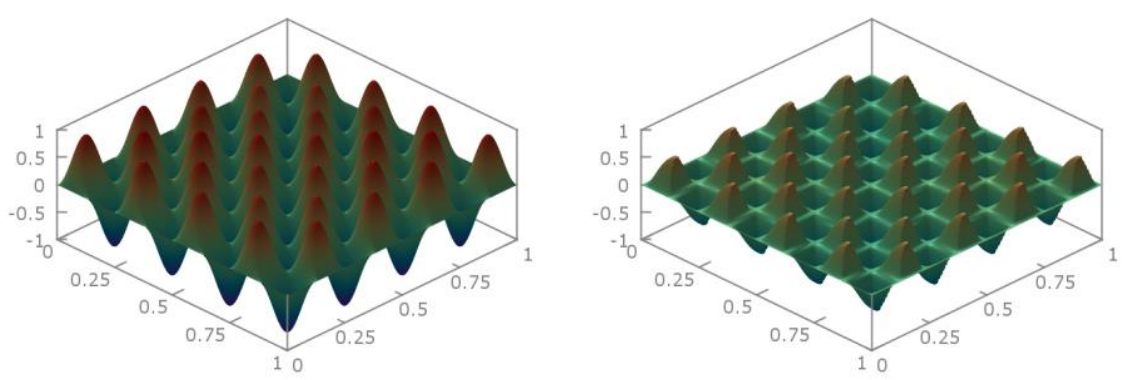

$$
t=0
$$
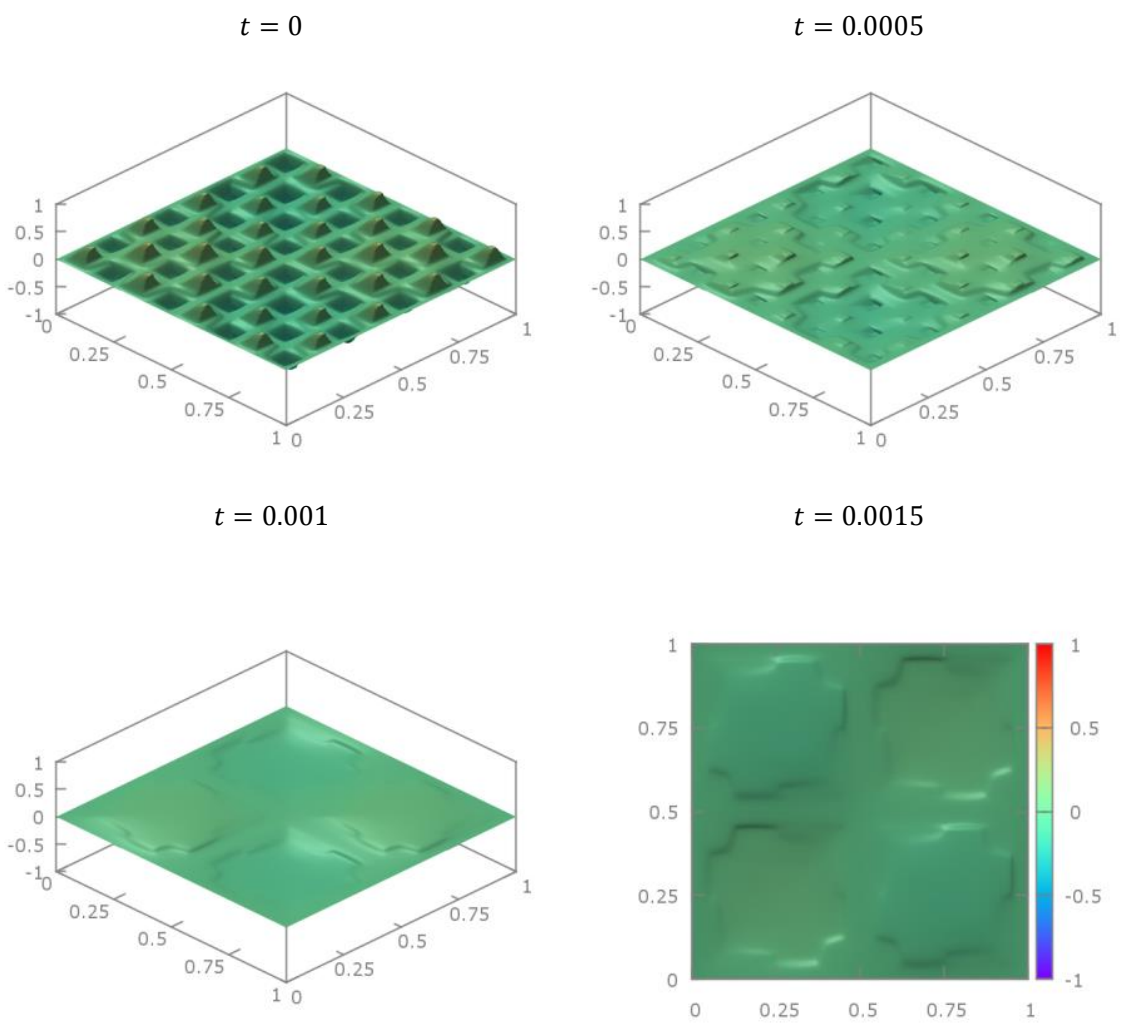

$$
t=0.002
$$

$$
t=0.002
$$

Figure 6

Morphology evolution for $f=0$, the regularized $l_{1}$ norm (13) with $A_{1}=0.01$, $p_{0}\left(x_{1}, x_{2}\right)=0.1 \sin \left(2 x_{1}\right) \sin \left(2 x_{2}\right)+0.9 \sin \left(4 x_{1}\right) \sin \left(4 x_{2}\right)$ at different times 

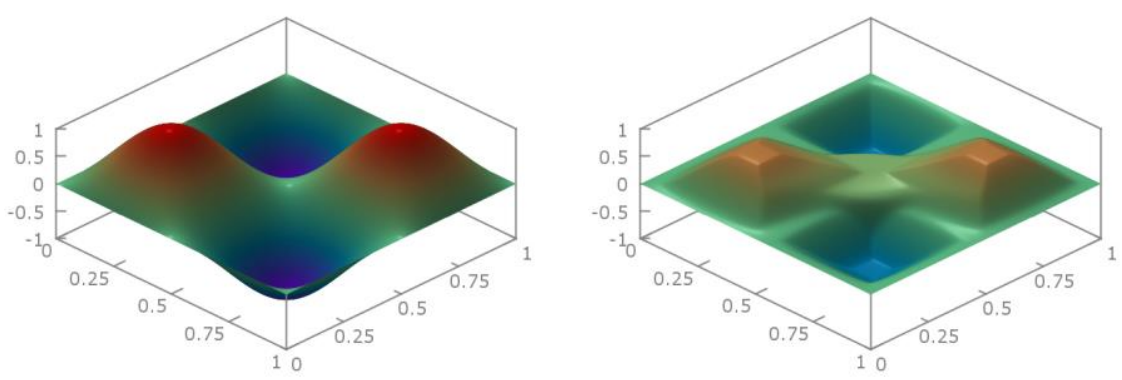

$t=0$

$$
t=0.006
$$
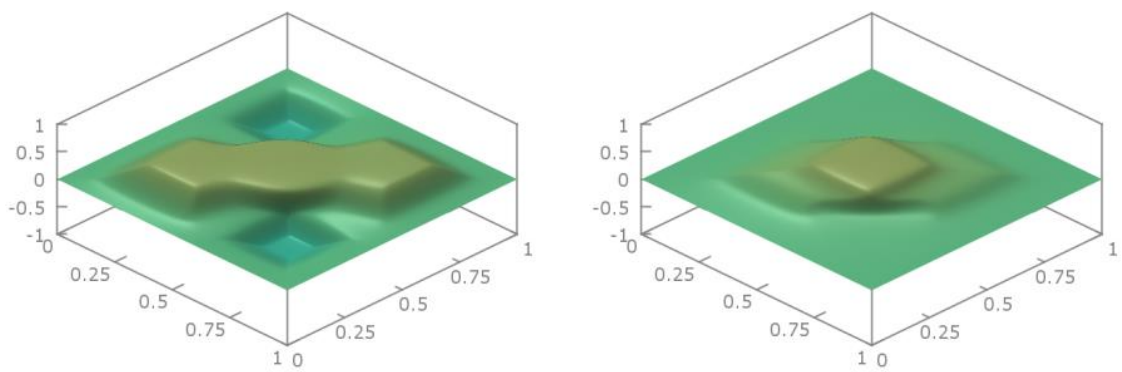

$$
t=0.012
$$

$$
t=0.02
$$
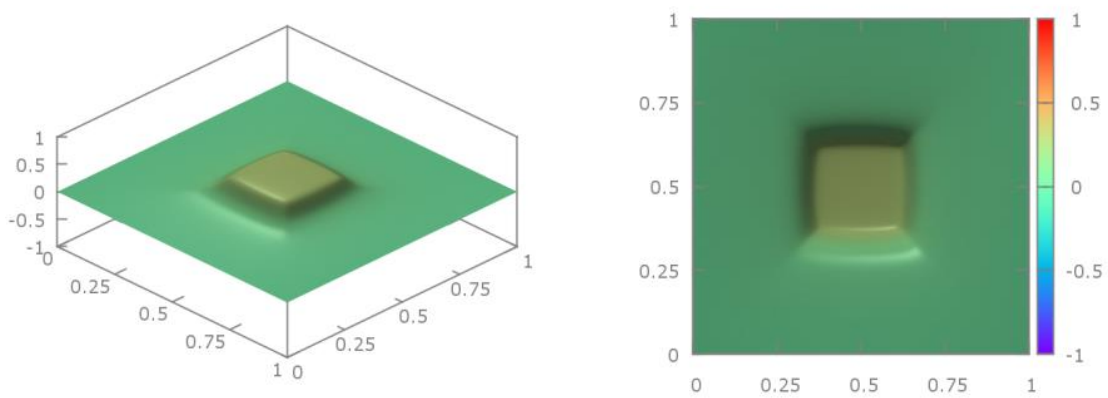

$$
t=0.03
$$

$$
t=0.03
$$

Figure 7

Morphology evolution for

$$
f=10\left(1-\tanh \left(20\left(\operatorname{sqrt}\left(\left(x_{1}-0.5\right)\left(x_{1}-0.5\right)+\left(x_{2}-0.5\right)\left(x_{2}-0.5\right)\right)-0.15\right)\right)\right),
$$

the regularized $l_{1}$ norm (13) with $A_{1}=0.01, p_{0}\left(x_{1}, x_{2}\right)=\sin \left(2 x_{1}\right) \sin \left(2 x_{2}\right)$ at different times 


\section{Conclusion}

In the paper, we have studied the anisotropic mean curvature flow in relative geometry for which a global existence result has been derived. A numerical scheme based on the method of lines has been presented and analysed concerning its stability. In the numerical experiments, the influence of various anisotropy symmetries and the forcing term on the surface evolution has been addressed.

\section{Acknowledgement}

This work was partially supported by the project No. P108/12/1463 "Two scales discrete-continuum approach to dislocation dynamics" of the Grant Agency of the Czech Republic.

\section{References}

[1] Bellettini, G., Paolini, M.: Anisotropic Motion by Mean Curvature in the Context of Finsler Geometry, Hokkaido Mathematical Journal, Vol. 25, No. 3, pp. 537-566, 1996

[2] Beneš, M.: Diffuse-Interface Treatment of the Anisotropic Mean-Curvature Flow, Applications of Mathematics, Vol. 48, pp. 437-453, 2003

[3] Beneš, M., Hilhorst, D., Weidenfeld, R.: Interface Dynamics for an Anisotropic Allen-Cahn Equation, in Nonlocal Elliptic and Parabolic Problems, pp. 39-45, eds. Biler P., Karch G. and Nadzieja T., Banach Center Publications, Volume 66, 2004, Institute of Mathematics, Polish Academy of Sciences, Warszawa, 2004

[4] Deckelnick, K., Dziuk, G.: Discrete Anisotropic Curvature Ow of Graphs, ESAIM: Mathematical Modelling and Numerical Analysis, Vol. 33, No. 6, pp. 1203-1222, 1999

[5] Deckelnick, K., Dziuk, G.: Error Estimates for a Semi-Implicit Fully Discrete Finite Element Scheme for the Mean Curvature Flow of Graphs, Interfaces and Free Boundaries, Vol. 2, No. 4, pp. 341-359, 2000

[6] Deckelnick, K., Dziuk, G.: Convergence of Numerical Schemes for the Approximation of Level Set Solutions to Mean Curvature Flow, Numerical Methods for Viscosity Solutions and Applications, Vol. 59, pp. 77-93, 2001

[7] Deckelnick, K., Dziuk, G.: A Fully Discrete Numerical Scheme for Weighted Mean Curvature Flow, Numerische Mathematik, Vol. 91, No. 3, pp. 423-452, 2002

[8] Dziuk, G.: Discrete Anisotropic Curve Shortening Flow, SIAM J. Numer. Anal., Vol. 36, No. 6, pp. 1808-1830, 1999

[9] Gurtin, M. E.: On the Two-Phase Stefan Problem with Interfacial Energy and Entropy, Archive for Rational Mechanics and Analysis, Vol. 96, No. 3, pp. 199-241, 1986 
[10] Haußer, F., Voigt, A.: A Numerical Scheme for Regularized Anisotropic Curve Shortening Flow, Applied Mathematics Letters, Vol. 19, No. 8, pp. 691-698, 2006

[11] Huisken, G.: Non-Parametric Mean-Curvature Evolution with BoundaryConditions, Journal of Differential Equations, Vol. 77, No. 2, pp. 369-378, 1989

[12] Lieberman, G. M.: The First Initial-Boundary Value Problem for Quasilinear Second Order Parabolic Equations, Annali della Scuola Normale Superiore di Pisa - Classe di Scienze, Vol. 13, No. 3, pp. 347-387, 1986

[13] Oberman, A. M.: A Convergent Monotone Difference Scheme for Motion of Level Sets by Mean Curvature, Numer. Math., Vol. 99, No. 2, pp. 365379,2004

[14] Pozzi, P.: Anisotropic Mean Curvature Flow for Two-Dimensional Surfaces in Higher Codimension: a Numerical Scheme, Interfaces and Free Boundaries, Vol. 10, No. 4, pp. 539-576, 2008

[15] Visintin, A.: Models of Phase Transitions, Birkhäuser, Boston, 1996 